\title{
Real-time in-situ Investigation of III-V Nanowire Growth using Custom-designed Hybrid Chemical Vapor Deposition-TEM
}

Kimberly Dick Thelander ${ }^{1,2}$, L. Reine Wallenberg ${ }^{2,3}$, Axel R. Persson ${ }^{2,3}$, Marcus Tornberg ${ }^{1}$, Daniel Jacobsson $^{3}$, Crispin Hetherington ${ }^{3}$, Joacim Gustafsson ${ }^{4}$, Stas Dogel ${ }^{5}$

1. Solid State Physics, Lund University, Lund, Sweden.

2. Center for Analysis and Synthesis, Lund University, Lund, Sweden

3. nCHREM, Lund University, Lund, Sweden

4. Spectral Solutions, Stockholm, Sweden

5. High-technology lab, Hitachi High-technology Canada, Rexdale, Ontario, Canada

Semiconductor nanowires are an important technology for applications in electronics, light harvesting and generation, and fundamental physics investigations [1]. Their advantages lay particularly in their geometry, with the nanoscale diameter allowing for quantum confinement and enhanced surface sensitivity, and their extended (axial) dimension greatly facilitating integration of the materials into complex structures and devices. However, of even greater importance is the potential for forming new, novel materials via the bottom-up crystal growth process in one dimension, with unusual properties that can be tuned according to design. These new materials include for example metastable crystal phases that do not form in bulk semiconductors, and metastable alloys of two or more semiconductors. Although these novel materials represent a great potential of semiconductor nanowires, the ability to develop and control their formation depends on a mechanistic understanding of the transient and dynamic processes that control their growth.

The in-situ growth of nanowires in environmental TEM offers insights into the growth processes unparalleled by any other technique. Since nanowire growth is normally performed in a precursor-based vapour phase atmosphere at high temperature, the dynamic processing controlling the formation of these structures cannot be directly deduced by analyzing only the final grown nanostructure. In particular, insitu TEM has given important insights into nucleation processes, morphology of the growth front, and transport pathways of the precursor species [2,3]. The in-situ TEM technique, with conventional semiconductor precursors gases introduced into a modified TEM, has recently been used to investigate the processes controlling crystal phase formation in $\mathrm{GaP}$ and $\mathrm{GaAs}$ nanowires. Two major breakthroughs have been demonstrated, in understanding the relationship between growth rate and crystal defects in GaP [4], and in understanding the role of the growth front geometry on the selection of crystal phase in GaAs [5].

The in-situ TEM technique clearly has enormous potential to provide major insights into crystal growth; however a major step in instrument capability is needed to fully realize this opportunity. In particular we require much better control of process conditions (temperature, pressure, gas flows), higher spatial resolution, and in-situ compositional information. At Lund University together with Hitachi we have recently designed and developed a unique in-situ TEM infrastructure specifically designed for controlled synthesis of III-V semiconductor materials, including but not limited to nanowires. The instrument combines an aberration-corrected environmental TEM with a custom-designed chemical vapor deposition (CVD) system, to produce a hybrid CVD-TEM infrastructure. 
The infrastructure is based around a $300 \mathrm{kV}$ Hitachi $3300 \mathrm{~S}$ Environmental TEM, with cold FEG emitter, heating stage, fast high-resolution cameras and an aplanatic B-COR image corrector provides space in the objective lens polepiece for a heating stage reactor with gas inlets, while still achieving an $86 \mathrm{pm}$ point resolution (in vacuum). The instrument also has scanning capability for real time analysis including EDX and secondary electron imaging capabilities together with conventional BF/DF STEM imaging. This is combined with a CVD system including full gas handling with mass flow control and concentration measurement for up to nine different gaseous reactants, including standard metalorganic and hydride semiconductor sources. Current design allows for in-situ deposition of nine semiconductors: GaAs, InAs, AlAs, GaP, InP, AlP, GaSb, InSb and AlSb. Reactive species are introduced to the sample via separate gas inlets with maximum pressure up to $10 \mathrm{~Pa}$, mimicking the typical reactor pressures using in conventional CVD. The gas handling system will allow switching between the different gases at will, or through programmed sequences.

Preliminary investigations are focusing on establishment of the processes for fabricating nanowires composed of each of the semiconductor species, in particular to understand how well growth parameters correlate with ex-situ growth ranges and establish the importance of any discrepencies. The aim is to deduce and account for any effects of the electron beam or of total pressure on global growth behavior. This will be facilitated by the use of an ex-situ growth chamber integrated with the CVD system, so that growth at equivalent conditions can be directly compared [6].

\section{References:}

[1] KA Dick, Prog. Cryst. Growth Char. Mater. 138 (2008), p. 138

[2] SH Oh et al., Science 330 (2010), p. 489

[3] BJ Kim et al., Science 322 (2008), p. 1070

[4] YC Chou et al., Science 343 (2014), p. 281

[5] D Jacobsson et al., Nature 531 (2016), p. 317

[6] The authors acknowledge funding from the Knut \& Alice Wallenberg Foundation, the Swedish Research Council, and NanoLund.
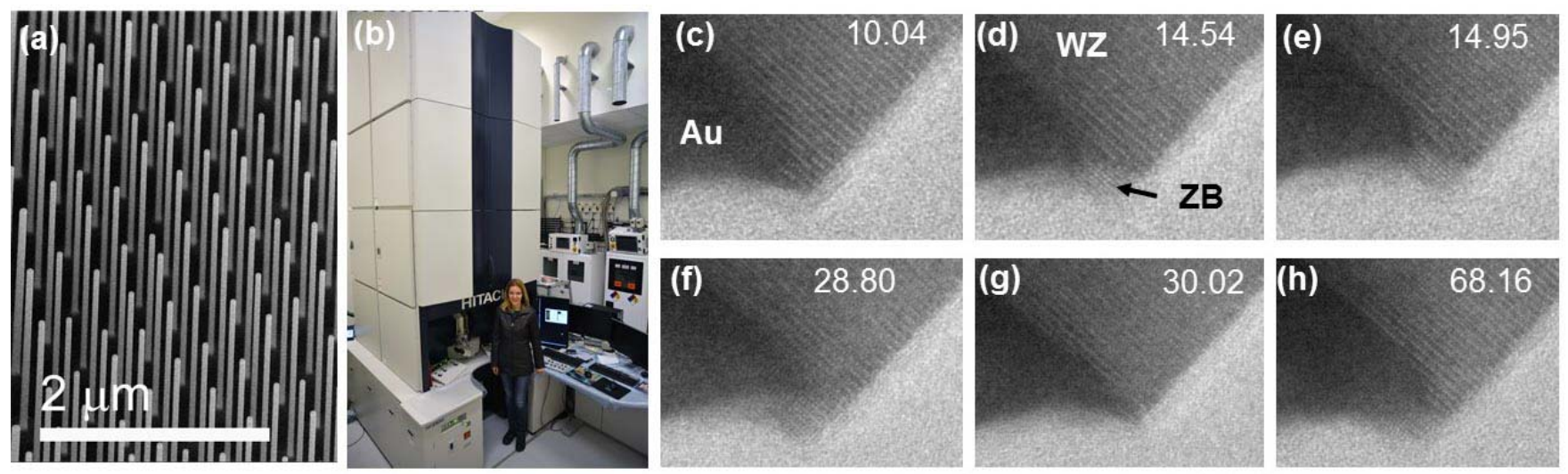

Figure 1. (a) Typical array of III-V nanowires grown by conventional CVD. (b) The custom CVDTEM installed in Lund. (c)-(h) Example image series illustrating the transformation of wurtzite crystal phase into zinc blende at elevated temperature in the TEM. Crystal phases are labelled in panel (d). The numbers indicate time stamps within the video in seconds. 